\title{
Seguridad remanente a flexión en forjados con corrosión en las viguetas
}

\section{Residual safety for flexural bending of slabs with corrosion in the joists}

E. Gil ${ }^{(*)}, \underline{\text { J. Vercher }^{(*)}}{ }^{\text {A. Mas }}{ }^{(*)}$, E. Fenollosa ${ }^{(*)}$

\section{RESUMEN}

A nivel nacional han aparecido un gran número de forjados de viguetas prefabricadas con graves problemas de corrosión, potenciada en muchas ocasiones por el empleo del cemento aluminoso. El propósito de este trabajo es evaluar la seguridad residual de los mecanismos de flexión en los casos más habituales de forjados dañados, para decidir la magnitud de la intervención necesaria. Los forjados dañados se han evaluado formando parte de un edificio completo, ya que las condiciones de contorno son fundamentales para la obtención del comportamiento real, teniendo en cuenta las distintas fases del proceso constructivo y de deterioro en el tiempo. En bastantes ocasiones en la inspección no se observa una fisuración importante en los elementos constructivos, como solados o tabiquería, y hemos comprobado que existe una seguridad residual suficiente para acometer las obras de rehabilitación sin necesidad de adoptar medidas excepcionales.

Palabras clave: Seguridad estructural remanente; corrosión de tendones; viguetas prefabricadas; prueba de carga.

\section{ABSTRACT}

A large number of slabs with severe corrosion problems at the precast joists have appeared in Spain, often increased by the use of aluminous cement. The purpose of this paper is to evaluate the residual safety for the bending mechanisms in the most common cases of damaged slabs, to decide the magnitude of the needed intervention. The damaged slabs have been evaluated as a part of an entire building, since the boundary conditions are essential for obtaining actual behavior, taking into account the different phases of the construction process and deterioration over time. A major cracking in the constructive elements are not frequently observed in inspections, and it is found that there is a sufficient residual safety to undertake the rehabilitation works without exceptional measures.

Keywords: Remaining structural safety; wire corrosion; precast joists; load test.

(*) Universitat Politècnica de València (España).

Persona de contacto/Corresponding author: jvercher@csa.upv.es (J. Vercher)

Cómo citar este artículo/Citation: Gil, E., Vercher, J., Mas, A., Fenollosa, E. (2015). Seguridad remanente a flexión en forjados con corrosión en las viguetas. Informes de la Construcción, 67(537): e054, doi: http://dx.doi.org/10.3989/ic.13.084.

Licencia / License: Salvo indicación contraria, todos los contenidos de la edición electrónica de Informes de la Construcción se distribuyen bajo una licencia de uso y distribución Creative Commons Reconocimiento no Comercial 3.o. España (cc-by-nc). 


\section{INTRODUCCIÓN}

Existen múltiples investigaciones con el propósito de conocer las causas y mecanismos de la corrosión del armado y para conocer las técnicas y materiales de reparación, pero se ha prestado muy poca atención a la evaluación de la integridad de estructuras corroídas. En esta investigación se evalúa la seguridad residual de los forjados con viguetas que tienen los tendones corroídos, habitualmente por empleo del cemento aluminoso, y se extraen conclusiones adecuadas que ayudan a decidir la fase de intervención.

La estimación de la seguridad residual de las estructuras tiene una importancia creciente, ya que cada vez es mayor el número de estructuras que se restauran o se reacondicionan, comparándolo con el número de estructuras que se construyen (1).

En nuestro país, las empresas constructoras de ámbito nacional afirman que la rehabilitación y el mantenimiento de edificios representan el 30\% de la actividad del sector de la construcción, con tendencia al alza, y se prevé que en los próximos años el presupuesto destinado al refuerzo de estructuras supere al destinado para las nuevas construcciones (2).

Pero, dentro del sector en alza de la rehabilitación, los elementos sometidos a flexión son los que más daños tienen. En España, aparecen lesiones en el 25,6\% de los forjados, y la corrosión en los forjados unidireccionales representa el $68 \%$ de las situaciones de patología de los mismos (3).

A nivel nacional han aparecido muchos casos de corrosión en los tendones inferiores de las viguetas, debido al uso del cemento aluminoso. Este cemento puede sufrir conversión, lo que da lugar a hormigones porosos, fácilmente atacables desde el exterior y con menos capacidad resistente (4). A nivel nacional, se estima, como más probable, que el número de viviendas en cuyos forjados se ha utilizado cemento aluminoso, y construidas en el periodo 1950-1970, alcanza un número comprendido entre 400.000 y 800.000 , de un total de unas 3.400.000 viviendas construidas en dicho periodo. El 25\% de las viguetas pretensadas utilizadas en la Comunidad Valenciana desde principio de los cincuenta hasta final de los setenta están fabricadas con cemento aluminoso (5).

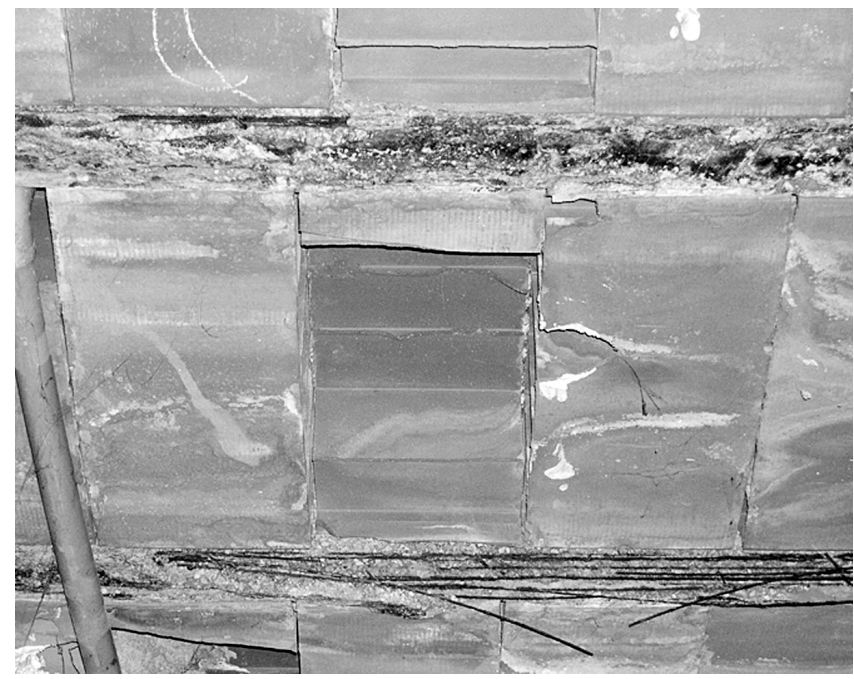

Figura 1. Corrosión severa en las viguetas de una vivienda en Benigánim (Valencia)
Dentro de nuestra dilatada experiencia en la evaluación de forjados dañados, una vez descartada la rotura por cortante, hemos encontrado en bastantes ocasiones casos reales en los que todos los tendones inferiores de algunas o incluso de todas las viguetas de un paño estaban totalmente corroídas. Y, sin embargo, no presentaba deformaciones excesivas ni daños de importancia en los elementos constructivos, como solados y tabiquería. Uno de los casos más llamativos es el caso del edificio sito en Benigánim (Valencia) de la Figura 1, en el que se descubrió el daño al demoler el falso techo debido a un cambio de uso del bajo comercial, y no se había notado ningún síntoma ni en esta planta ni en la superior. En esta figura se aprecia el desprendimiento completo del recubrimiento y que el armado inferior de las viguetas está cortado debido a la corrosión. Únicamente se puede ver la marca del óxido dejada por los tendones en las viguetas.

La principal dificultad frente a la que se encuentran los técnicos al analizar los casos de patología estructural es evaluar la seguridad remanente y los criterios de aceptación necesarios para aconsejar el tipo de medidas a adoptar, desde pequeñas medidas de protección hasta el apuntalamiento, desalojo, o incluso el derribo. Este trabajo se centra en este aspecto, y propone una metodología adecuada para poder cuantificar y decidir la actuación.

No existe a nivel nacional ni internacional una normativa específica que trate sobre el diseño y cálculo de las actuaciones de reparación y refuerzo sobre las estructuras de edificación. Entre los escasos textos publicados por un organismo oficial, a nivel nacional existe la «Reparación y refuerzo de estructuras de hormigón. Guía FIP de buena práctica», editado por el Grupo Español del Hormigón (6). A nivel autonómico, el Instituto Valenciano de la Edificación ha desarrollado unas guías de inspección, evaluación en intervención (4) (5). Pero en todas estas publicaciones de organismos oficiales se trazan ligeramente las pautas a seguir en caso de enfrentarse a una patología estructural en el hormigón armado, pero no se establecen reglas de diseño ni método de cálculo alguno, siendo estas normativas en ocasiones contradictorias.

La primera tarea que debe realizar un experto cuando perite un forjado con daños es descartar el fallo por cortante, debido a su peligrosidad. Se debe estudiar el detalle constructivo real del encuentro entre la jácena y la vigueta, comprobando al menos los siguientes aspectos: la existencia de una zona de macizado o de una improbable armadura, pasador de cortante, la anchura y estado del alma de la vigueta, la anchura del hormigón in situ y su adherencia con el prefabricado, y el estado de la armadura de negativos, que controla la fisuración del hormigón en la parte superior del forjado. Los desplomes instantáneos de los forjados siempre han aparecido asociados a fallos por cortante en los bordes de los paños. En los casos analizados en los que ha habido problemas de cortante, los nervios poseían almas muy finas con escaso hormigón de relleno a su alrededor, entre la bovedilla y la vigueta.

Este estudio se centra en la evaluación de la seguridad residual existente en los casos de forjados con corrosión cuya rotura está dominada por la flexión, que suelen ser los más habituales, porque la corrosión aparece en las armaduras inferiores de los nervios del forjado. Esto es debido a la menor protección que posee este armado inferior frente al superior, en cuanto a recubrimientos se refiere (Figura 2). 


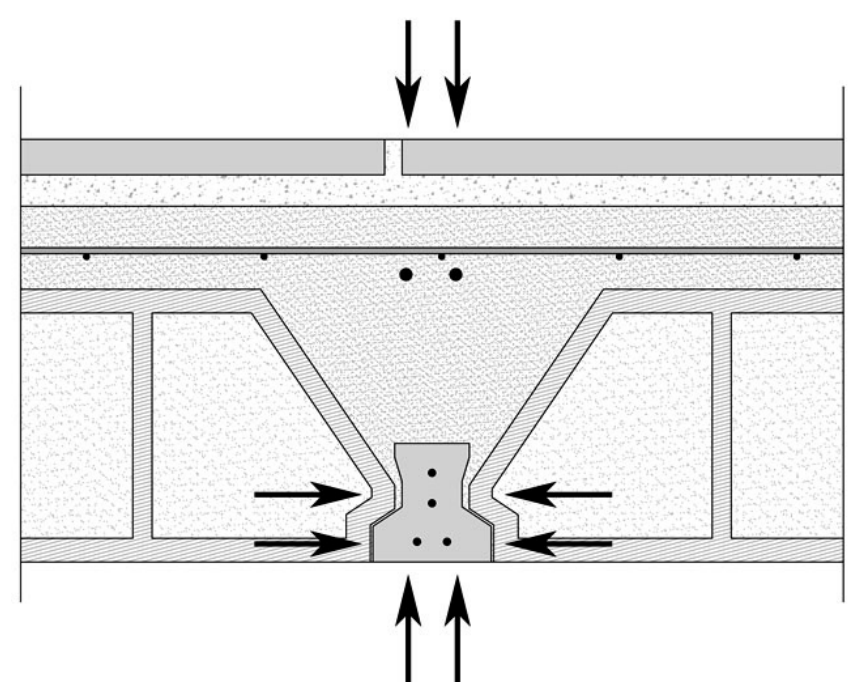

Figura 2. Frentes de ataque de las acciones externas.

Se analiza la seguridad residual de una gran variedad de casos de forjados habituales, ejecutados con viguetas prefabricadas pretensadas y que presentan corrosión en los tendones inferiores, estudiando:

- Cantos de forjado de 25 y $30 \mathrm{~cm}$.

- Grado de corrosión y niveles de tendones sanos.

- Número de viguetas afectadas.

- Posición de viguetas afectadas en el forjado.

- Calidades de los materiales y su deterioro.

- Desprendimiento del recubrimiento inferior del prefabricado.

Los resultados extraídos sirven para ayudar a decidir en la gran mayoría de casos de forjados de edificación. Así mismo, la metodología empleada puede utilizarse en otros casos más singulares.

\section{ANÁLISIS DE LA SEGURIDAD REMANENTE EN FORJADOS CON TENDONES CORROÍDOS EN LAS VIGUETAS}

En el campo de la edificación no se han realizado grandes esfuerzos por llegar a evaluar de forma precisa la seguridad remanente en casos de corrosión, sino que se ha reparado o reforzado, dejando la construcción existente del lado de la seguridad. Los escasos estudios encontrados al respecto se centran en el análisis de elementos simples sometidos a flexión (7) (8).

Una estructura puede encontrar en el espacio otros mecanismos, diferentes a las hipótesis habitualmente adoptadas en los modelos de estructuras de barras. La simplificación del comportamiento de barra identificada además por una única línea, su línea neutra, no consigue describir el balance de trabajo mínimo con el que se comporta en la realidad una estructura. Aparecen otras formas de trabajo, como el arco (8) o el efecto membrana (9), la formación de bielas y tirantes y la transmisión de cargas en el espacio.

Además, se ha demostrado que el forjado trabaja en conjunto, ya que en algunos casos peritados se han extraído nervios aislados y se han ensayado a flexión pura, obteniendo resultados respecto a la seguridad muy inferiores a los que se tienen en el forjado completo. Por este motivo la investi- gación se realiza en modelos de edificio completo tridimensionales donde el forjado posee las condiciones de contorno reales.

La metodología propuesta para la evaluación de la seguridad remanente de un forjado de viguetas prefabricadas con corrosión severa se basa en dos aspectos. Por un lado, se emplea el criterio de aceptación mediante prueba de carga propuesta por la EHE-08. Es necesario realizar una prueba de carga cuya carga total (incluyendo el peso propio presente) no debe ser inferior a $0,85(1,35 G+1,5 Q)$, donde $G$ es la suma de los pesos propios y $Q$ es la suma de las sobrecargas. Las deformaciones medidas deben satisfacer la ecuación [1], donde $l$ es la luz de las viguetas y $h$ el espesor del forjado:

$$
\Delta_{1} \leq \frac{l^{2}}{20000 h}
$$

Si la deformación es mayor, se debe medir la flecha recuperada tras la descarga. La flecha remanente debe ser inferior al $25 \%$ en elementos armados, e inferior al $20 \%$ en elementos pretensados. En la Tabla 1 se puede ver el valor de prueba de carga y de incremento de deformada límite para los diferentes cantos de forjado estudiados.

Y, una vez se ha comprobado que el forjado puede permanecer en uso, se analiza el Factor de Carga (FC). Este factor de carga está propuesto en esta investigación, y relaciona la carga a la cual cada espécimen alcanza la flecha activa admisible respecto a la tabiquería según EHE con la carga de norma, suma de las concargas y las sobrecargas. Con el estudio de este factor se tiene una estimación de la magnitud de la intervención necesaria, con lo que se ayuda al experto responsable.

\subsection{Geometría de los casos estudiados}

El modelo empleado en este trabajo es un edificio genérico de viviendas de 3 plantas y 3 vanos en ambas direcciones, con luces y alturas habituales. Como se ha indicado previamente, se han estudiado los forjados unidireccionales de viguetas prefabricadas con los cantos más habituales, 25 y $30 \mathrm{~cm}$.

Los modelos de forjado estudiados son tridimensionales y están insertados dentro de un edificio completo, con su estructura de hormigón armado y su fachada cerámica, simuladas de forma precisa. De este modo poseen unas condiciones de contorno muy realistas, y pueden aparecer los mecanismos de resistencia adicional, que son cruciales en la estabilidad de un forjado con daños.

En la Figura 3 puede verse la geometría del cuarto del edificio que ha sido simulado, ya que se ha aprovechado la doble simetría para reducir el coste computacional.

La estructura del edificio se compone de pórticos de vigas planas y viguetas prefabricadas pretensadas, sobre las que apoya la capa de compresión. Todos los elementos estructurales poseen secciones y armados habituales. La altura libre entre forjados es de 2,64 m. Los pilares tienen una sección cuadrada de $30 \mathrm{~cm} \times 30 \mathrm{~cm}$, y su armado está compuesto por $4 \varnothing 12$ y estribos de Ø6 cada $24 \mathrm{~cm}$. Las luces a ejes de pilares, tanto en la dirección de las vigas como en la de las viguetas, son del entorno de los $5 \mathrm{~m}$. Las vigas planas poseen el canto del forjado y son de $60 \mathrm{~cm}$ de ancho. El refuerzo de positivos 


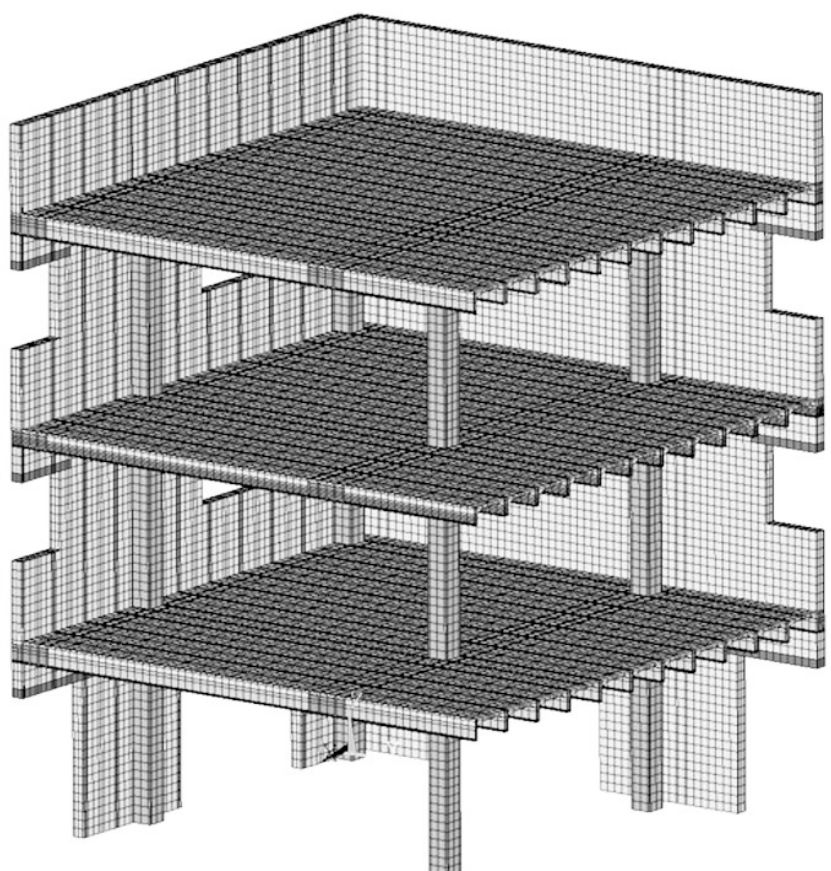

Figura 3. Geometría de un cuarto del edificio (elementos finitos).

lo componen $3 \varnothing_{12}+4 \varnothing_{16}$, y el de negativos $3 \varnothing_{12}+4 \varnothing_{20}$, para los casos de forjados de $30 \mathrm{~cm}$ de canto. En los casos de $25 \mathrm{~cm}$ de canto, se colocan $7 \varnothing_{20}$ tanto para momentos positivos como negativos. Las vigas poseen estribos de 4 ramas de Ø6 con una separación variable. La separación entre viguetas es de $69 \mathrm{~cm}$. Se han utilizado dos tipos de viguetas elaboradas por Prevalesa SL (10), con la intención de evaluar la influencia del número de niveles de tendones pretensados de las viguetas prefabricadas. Las viguetas elegidas son la $\mathrm{T}$ 12.2 y la $\mathrm{T} 12.3$, que son la misma vigueta con 2 niveles $\left(2 \varnothing_{5}+\right.$ 1Ø5) y 3 niveles $\left(2 \varnothing_{5}+1 \varnothing_{5}+1 \varnothing_{5}\right)$ de tendones pretensados respectivamente. En la capa de compresión se ha introducido un mallazo de reparto compuesto por $\varnothing_{5}$ cada $25 \mathrm{~cm}$ en ambas direcciones.

La fachada cerámica se apoya en los forjados y se compone de dos hojas con una cámara de aire entre ellas. Los huecos son de dimensiones convencionales en edificios de viviendas.

\subsection{Análisis en el tiempo del proceso constructivo y de deterioro}

Con la intención de que los resultados y las conclusiones extraídas se aproximen al máximo al comportamiento real del edificio, se han tenido en cuenta las distintas fases en el proceso constructivo y el daño se ha simulado en el momento en que aparece en la realidad.

El software de elementos finitos Ansys permite crear una serie de pasos de carga. En cada paso aparecen o desaparecen los elementos deseados mediante la opción de nacimiento y muerte de elementos. De este modo, cada elemento ejerce influencia desde el momento real en que aparece en la construcción y aporta rigidez. Además, en cada paso se aplica la carga deseada. Debido a que se evalúan dos cantos de forjado, 25 y $30 \mathrm{~cm}$, los pasos de carga son distintos, y se resumen en la Tabla 1. De este modo, los pasos realizados son: a) Viguetas pretensadas. El pretensado se aplica mediante fuerzas de pretensado equivalentes, que se obtienen empleando las hojas de autorización de uso, en este caso de la empresa Prevalesa SL (10). El pretensado inicial es de $1.255 \mathrm{~N} / \mathrm{mm}^{2}$ y se espera una pérdida de hasta el $29 \%$ a lo largo del tiempo. Por tanto, se aplica una fuerza de pretensado equivalente de $17.000 \mathrm{~N}$ por cada redondo de $\varnothing$ 5. En el primer paso aparecen solo las viguetas pretensadas ocupando su posición en el forjado.

b) Estructura de hormigón armado. Posteriormente aparece el resto de la estructura de hormigón armado con su peso propio.

c) Solado + falso techo. Estos elementos aparecen en este momento y se simulan con un incremento de carga sobre los forjados de $1.000 \mathrm{~N} / \mathrm{m}^{2}$.

d) Fachada + tabiquería. La fachada cerámica aparece con su peso propio, rigidizando y colaborando en la estabilidad de los forjados. Por otro lado, la tabiquería se introduce como una carga de $1.000 \mathrm{~N} / \mathrm{m}^{2}$ sobre los forjados. A partir de este momento se produce la flecha activa respecto a la tabiquería, que está limitada al valor de $L / 400$ por EHE-08, siendo $L$ la luz en metros. Como la geometría estudiada tiene una luz de viguetas a ejes de pilares de 4,92 m, la flecha activa admisible es de $1,23 \mathrm{~cm}$.

e) Carga de servicio. Es la carga a la que se encuentra sometido el forjado a lo largo de su vida útil. Está compuesta por la totalidad de las concargas más una parte de la sobrecarga de uso, según CTE-06 (11). El forjado entero no está sobrecargado con el valor total de la sobrecarga de uso todo el tiempo. Se emplea el valor cuasipermanente de esta sobrecarga, que es el valor de sobrecarga que se excede durante el $50 \%$ del tiempo de referencia. Con una carga sobre los forjados de valor igual a la carga de servicio aparecen los daños por corrosión, ya que lo hacen en la vida en servicio del edificio.

f) Corrosión. En un sexto paso de carga se representan los daños ocasionados por la corrosión. Con la intención de poseer unas condiciones de contorno intermedias ideales se simulan los daños en el segundo forjado. Como este trabajo se centra en los casos patológicos más extremos, cuando aparece la corrosión en el armado inferior de una vigueta, estos redondos desaparecen y se pierde completamente el recubrimiento inferior. Los niveles de tendones superiores se mantienen sanos. Los casos en que aparezca la corrosión no tan grave, están del lado de la seguridad teniendo en cuenta las conclusiones y recomendaciones desarrolladas en este estudio.

Tabla 1. Pasos de carga para los diferentes cantos de forjados.

\begin{tabular}{|c|c|c|}
\hline $\operatorname{Cargas}\left(\mathrm{N} / \mathbf{m}^{2}\right)$ & $30 \mathrm{~cm}$ & $25 \mathrm{~cm}$ \\
\hline Peso propio del forjado & 3.500 & 2.750 \\
\hline Solado + falso techo & 1.000 & 1.000 \\
\hline Tabiquería & 1.000 & 1.000 \\
\hline PESO PROPIO & $5 \cdot 500$ & 4.750 \\
\hline Sobrecarga de uso cuasipermanente & 600 & 600 \\
\hline Carga de servicio & 6.100 & $5 \cdot 350$ \\
\hline Sobrecarga de uso & 2.000 & 2.000 \\
\hline CARGA DE NORMA & $7 \cdot 500$ & 6.750 \\
\hline \multicolumn{3}{|l|}{ Prueba de carga EHE } \\
\hline Carga & 8.861 & 8.000 \\
\hline Inc. deform. admisible & $4,03 \mathrm{~mm}$ & $4,84 \mathrm{~mm}$ \\
\hline
\end{tabular}


g) Sobrecarga total. A continuación, con determinados nervios dañados, se aplica la totalidad de la sobrecarga de uso $\left(2.000 \mathrm{~N} / \mathrm{m}^{2}\right)$. La edificación cumpliría la normativa con un coeficiente de seguridad 1 al estar cargada con 7.500 y $6.750 \mathrm{~N} / \mathrm{m}^{2}$ en los forjados de 30 y $25 \mathrm{~cm}$ de canto respectivamente. Este es el valor de concargas más sobrecargas, y se ha denominado como carga de norma.

h) Carga hasta la rotura. Para finalizar, se carga el forjado en las zonas dañadas hasta que colapsa.

\subsection{Propiedades de los materiales}

El modelo de edificio está compuesto por tres materiales, el hormigón, el acero de refuerzo y la fachada cerámica. Debido a que las viguetas son prefabricadas y el resto de la estructura de hormigón armado está construida in situ, aparecen distintas calidades tanto de acero como de hormigón. Las viguetas prefabricadas están formadas por hormigón HP-40 y acero de pretensar Y-1860-C (10).

Con la intención de que este estudio sirva al mayor número de casos posibles, se analizan los casos de hormigón in situ de buena (HA-30) y baja calidad (HA-17,5). De este modo, realizando un ensayo de la resistencia del hormigón in situ en el edificio real, se pueden extraer directamente conclusiones.

En el caso del acero se analizan también varias calidades, el acero B-400-S y el B-500-SD.

Para abarcar los edificios en los que la conversión del cemento aluminoso haya hecho aparición, se analizan casos con un hormigón dañado, con unas características resistentes muy mermadas, el HA-10.

El técnico responsable debe identificar el tipo de vigueta y la calidad de los materiales empleados. En esta investigación se analizan dos categorías de materiales, además de una tercera que corresponde a un hormigón muy deteriorado por aparición de la conversión en toda la estructura (HA-10).

El hormigón posee un comportamiento multilineal isotrópico (12) y se emplea el criterio de rotura espacial de Willam-Warnke (13). Unas características muy importantes para simular el correcto comportamiento del hormigón son los coeficientes de transferencia por cortante. Cuantifican, en tanto por 1 , la parte del esfuerzo cortante que pasa de una cara a la otra de una fisura, para los casos de fisura abierta y cerrada. Se ha realizado una labor exhaustiva para calibrar estos coeficientes (14). Se han utilizado unos valores de 0,15 y o,6 para fisura abierta y cerrada respectivamente.

Para la obtención de las resistencias a tracción y a compresión del hormigón se ha empleado la norma EH-91, debido al empleo de esta normativa de cálculo en la mayoría de edificios que son actualmente peritados.
El comportamiento del acero se puede simplificar con una curva bilineal isotrópica.

Y la fachada cerámica es un material heterogéneo, ya que contiene ladrillos cerámicos y juntas de mortero, que son planos de debilidad. Su comportamiento, al igual que en el caso del hormigón, es multilineal. Se ha optado por una macromodelización, representando la cerámica y el mortero como un material único.

La resistencia a compresión se ha obtenido del Documento Básico de Seguridad Estructural en la Fábrica (DB SE-F) del CTE-06. El resto de propiedades se han extraído de los estudios realizados por Cubel y otros (15), Brencich y de Felice (16), y Dilrukshi y otros (17).

La Tabla 2 resume las propiedades de los materiales empleados.

\subsection{Validación de los modelos}

Debido a las dimensiones y a la complejidad de los modelos evaluados, no es posible realizar ensayos reales para contrastar los resultados obtenidos con las simulaciones. Por otro lado, no se han encontrado investigaciones similares realizadas por otros autores en la bibliografía relacionada. Por todo esto, la forma en que se ha validado la metodología es mediante la simulación de elementos simples sometidos a los distintos tipos de solicitaciones, encontrados en publicaciones de otros autores.

Se han reproducido ensayos de elementos simples de hormigón armado sometidos a flexión (18) y a compresión (19), obteniendo en todos ellos resultados acordes.

Ya que el aspecto que gobierna el colapso en esta investigación es la corrosión en algunas viguetas, la validación de elementos simples con corrosión sometidos a flexión es esencial. Se ha reproducido el comportamiento de los elementos de hormigón con diferentes grados de corrosión en su armadura ensayados por Rodríguez y otros (GEOCISA-Dragados Group) (20). Estos elementos ya fueron simulados con el software de elementos finitos Ansys por Coronelli y Gambarova (8). Se ha obtenido una gran concordancia en las curvas cargadesplazamiento en todos los casos. En la Figura 4 se muestra el caso 11.4, como ejemplo ilustrativo. Este espécimen tiene $2 \varnothing_{10}$ como armadura inferior y $2 \varnothing 8$ como armadura superior, además de estribos de Ø6 cada $170 \mathrm{~mm}$. Se le induce una corrosión del 17,2\% en las barras inferiores, significando esto un ataque de $0,45 \mathrm{~mm}$, una corrosión del $24,3 \%$ en las barras superiores, que son $0,52 \mathrm{~mm}$, y una corrosión en los estribos del 24,3\%, o lo que es lo mismo o,39 mm. Coronelli y Gambarova simularon la corrosión reduciendo las secciones y las propiedades tanto del hormigón como del acero. Se ha conseguido una correspondencia en el comportamiento de

Tabla 2. Propiedades de los materiales.

\begin{tabular}{|c|c|c|c|c|c|c|c|c|}
\hline \multirow{2}{*}{ MATERIAL } & \multicolumn{4}{|c|}{ HORMIGÓN } & \multicolumn{3}{|c|}{ ACERO } & \multirow{2}{*}{ FÁBRICA } \\
\hline & HA-10 & HA-17,5 & HA-30 & HP-40 & B-400-S & B-500-SD & Y-1860-C & \\
\hline $\mathbf{f}_{\mathrm{cm}}(\mathbf{M P a})$ & 12,75 & 22 & 36,28 & 48,05 & - & - & - & 4 \\
\hline E (GPa) & 9,04 & 15,15 & 28,6 & 30,9 & 200 & 200 & 200 & 2 \\
\hline $\mathbf{f}_{\mathrm{tm}}(\mathbf{M P a})$ & 0,95 & 1,41 & 1,98 & 2,40 & - & - & - & 0,2 \\
\hline$f_{y}(M P a)$ & - & - & - & - & 400 & 500 & 1.581 & - \\
\hline
\end{tabular}




\section{Carga (N)}

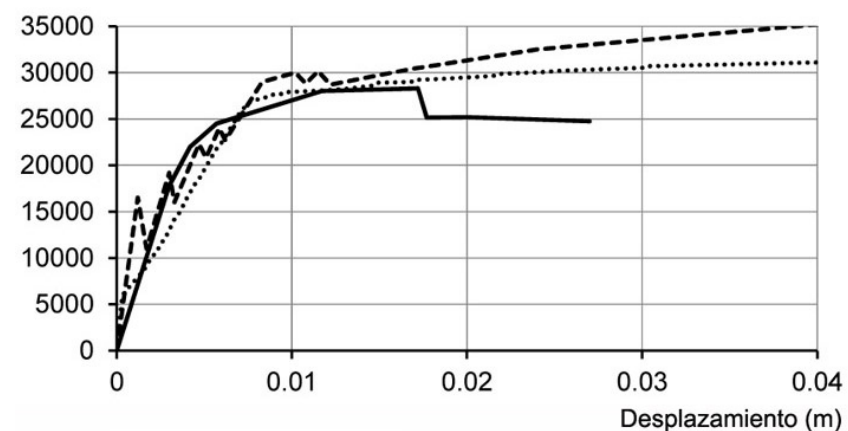

- Rodriguez y otros

---Coronelli y Gambarova

......Simulación propia

Figura 4. Curvas carga-desplazamiento del especímen 11.4 en los ensayos de Rodríguez y otros, Coronelli y Gambarova, y nuestra simulación.

los distintos modelos siguiendo sus especificaciones a la hora de realizar las simulaciones.

Pero los casos que se abordan en nuestra investigación, los que la han motivado, son los casos patológicos más severos, en los que el grado de corrosión es tal que se pierde completamente el recubrimiento y se cortan los tendones inferiores debido a la corrosión. En estos casos extremos, tanto el hormigón del recubrimiento como el armado inferior no colaboran en la sustentación, quedándose el hormigón y los tendones restantes como únicos elementos que resisten.

Debido a esto, en los primeros pasos de carga, consecuentes con los tiempos de construcción, se considera toda la vigueta sana. Y, en el último estado, la armadura corroída desaparece completamente, por corresponder esto con los casos estudiados y con muchos otros casos de daño en que la oxidación es parcial, pero se ha perdido totalmente la adherencia del tendón con el hormigón.

\section{CASOS ESTUDIADOS Y RESULTADOS OBTENIDOS}

El estudio comprende una gran campaña de modelos con forjados con los cantos más habituales, centrándonos en el forjado de $25 \mathrm{~cm}$ de canto, por su amplio empleo en España, y el forjado de $30 \mathrm{~cm}$ de canto, para tener un orden de interpolación con posibles variaciones del canto.

Por otro lado, se analiza la influencia del número de niveles de tendones pretensados que poseen las viguetas prefabricadas.

Se han simulado especímenes con distintas categorías de materiales: materiales de buena calidad (HA-30 y B-500-SD), materiales de menor calidad (HA-17,5 y B-400-S) y materiales dañados (HA-10 y B-400-S), posiblemente por conversión del cemento aluminoso tanto en las viguetas como en la estructura en general. El experto debe averiguar las propiedades que poseen los materiales del edificio que está peritando. El hormigón HA-17,5 y el acero B-400-S se emplearon ampliamente en la etapa de construcción masiva en nuestro país, durante los años 50-70, y el hormigón HA-30 y el acero B-500-SD se utilizan habitualmente en la actualidad. El estudio de casos con materiales de baja calidad y buena calidad permite realizar también una interpolación de resultados en caso de encontrar unas propiedades intermedias.
Se incluyen casos con un número variable de viguetas afectadas en las distintas posiciones de un forjado, todos ellos con desprendimiento total del recubrimiento inferior de hormigón. En la Figura 5 se representa la nomenclatura de paños, cuadrantes y puntos singulares de la geometría analizada. Los cuadrantes tipo son: 1, el cuadrante en esquina; 2, el cuadrante con continuidad de viguetas por un extremo; 3 , el cuadrante con continuidad de vigas por un extremo; y 4, el cuadrante central. Los puntos singulares son los puntos centrales de cada uno de los cuadrantes tipo. En cada uno de los casos se simula la prueba de carga de la EHE y se obtienen las curvas carga-desplazamiento en los puntos singulares, y en estas curvas se analiza la seguridad residual.

Por motivos de brevedad se exponen, como ejemplo, los resultados de los cuadrantes 2 y 4 en la Tabla 3, y se representan unas de las curvas a modo ilustrativo en las Figuras 6 y 7 . En la Tabla 3 se describen todos los casos evaluados, así como los valores de Factor de Carga y cumplimiento de prueba de carga para cada caso.

En las curvas carga-desplazamiento vertical se pueden observar una serie de aspectos, que son los siguientes:

- La contraflecha inicial producida por el proceso de prefabricado.

- Un pequeño escalón producido por la entrada de la tabiquería.

- El peldaño producido por la corrosión a la carga de servicio, con la carga de uso cuasipermanente. El final de este escalón es el punto de partida para la simulación de la prueba de carga de aceptación de estructuras existentes propuesta por la EHE.

- Se acota el incremento de deformación permitido para las curvas extremas, la más favorable y la más desfavorable.

- En el caso más desfavorable, con hormigón de 10 MPa y un solo nivel de tendones sanos, se ha simulado el proceso de descarga propuesto como segunda posibilidad de aceptación. La deformada ha sido superior al primer criterio y la

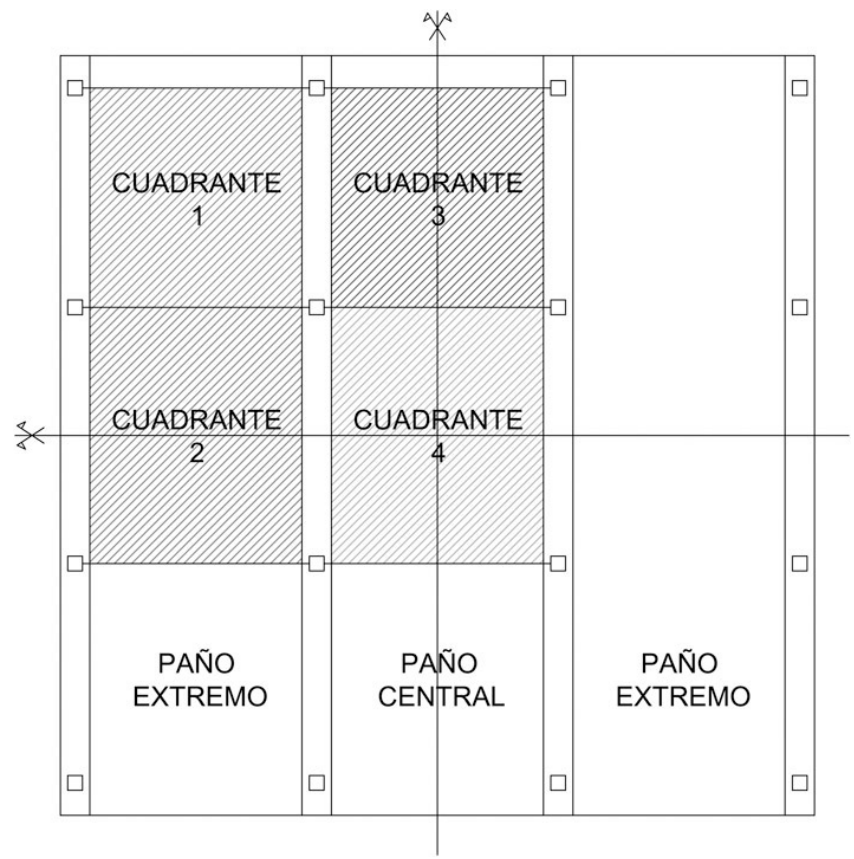

Figura 5. Nomenclatura de paños y cuadrantes del modelo estudiado. 
Tabla 3. Factor de Carga y cumplimiento de la prueba de carga de la EHE para los diferentes casos.

\begin{tabular}{|c|c|c|c|c|c|c|c|}
\hline \multicolumn{5}{|c|}{ Forjados de $25 \mathrm{~cm}$ de canto } & \multirow{2}{*}{$\begin{array}{c}\text { Carga a la } \\
\text { flecha activa } \\
\text { admisible } \\
\left(\mathbf{N} / \mathbf{m}^{2}\right)\end{array}$} & \multirow[b]{2}{*}{$\begin{array}{l}\text { Factor de Carga } \\
\mathbf{Q}_{\text {fl.act.adm. }} / \mathbf{Q}_{\text {norma }}\end{array}$} & \multirow[b]{2}{*}{$\begin{array}{l}\text { Prueba de } \\
\text { Carga EHE }\end{array}$} \\
\hline Cuadrante & $\begin{array}{c}\mathrm{N}^{\circ} \\
\text { viguetas } \\
\text { afectadas }\end{array}$ & $\begin{array}{c}\text { Materiales } \\
\text { (h.in situ - acero - } \\
\text { h.pretensado) }\end{array}$ & $\begin{array}{l}\text { Niveles } \\
\text { tendones } \\
\text { sanos }\end{array}$ & Caso & & & \\
\hline \multirow{16}{*}{2} & \multirow{4}{*}{1} & HA-17,5 - B-40O-S - HP-4O & 1 & A.1.1. & 10.554 & 1,56 & CUMPLE \\
\hline & & & 2 & A.1.2. & 12.001 & 1,78 & CUMPLE \\
\hline & & HA-30 - B-50O-SD - HP-40 & 1 & A.1.3. & 12.845 & 1,90 & CUMPLE \\
\hline & & & 2 & A.1.4. & 14.312 & 2,12 & CUMPLE \\
\hline & \multirow{8}{*}{3} & HA-10 - B-40O-S - HP-4O & 1 & A.2.1. & 8.216 & 1,22 & - NO - \\
\hline & & & 2 & A.2.2. & 9.641 & 1,43 & CUMPLE \\
\hline & & HA-17,5 - B-400-S - HP-30 & 1 & A.2.3. & 9.515 & 1,41 & CUMPLE \\
\hline & & & 2 & A.2.4. & 11.034 & 1,63 & CUMPLE \\
\hline & & HA-17,5 - B-400-S - HP-40 & 1 & A.2.5. & 9.659 & 1,43 & CUMPLE \\
\hline & & & 2 & A.2.6. & 11.107 & 1,65 & CUMPLE \\
\hline & & HA-30 - B-50O-SD - HP-40 & 1 & A.2.7. & 11.845 & 1,75 & CUMPLE \\
\hline & & & 2 & A.2.8. & 13.536 & 2,01 & CUMPLE \\
\hline & \multirow{4}{*}{ Todas } & HA-17,5 - B-40O-S - HP-40 & 1 & A.3.1. & 7.452 & 1,10 & - NO - \\
\hline & & & 2 & A.3.2. & 9.047 & 1,34 & CUMPLE \\
\hline & & HA-30 - B-50O-SD - HP-40 & 1 & A.3.3. & 9.260 & 1,37 & - NO - \\
\hline & & & 2 & A.3.4. & 11.027 & 1,63 & CUMPLE \\
\hline \multirow{16}{*}{4} & \multirow{4}{*}{1} & HA-17,5 - B-40O-S - HP-4O & 1 & B.1.1. & 11.082 & 1,64 & CUMPLE \\
\hline & & & 2 & B.1.2. & 12.496 & 1,85 & CUMPLE \\
\hline & & HA-30 - B-5OO-SD - HP-4O & 1 & B.1.3. & 13.946 & 2,07 & CUMPLE \\
\hline & & & 2 & B.1.4. & 15.595 & 2,31 & CUMPLE \\
\hline & \multirow{12}{*}{ Todas } & HA-10 - B-40O-S - HP-4O & 1 & B.2.1. & 8.545 & 1,27 & - NO - \\
\hline & & & 2 & B.2.2. & 9.880 & 1,46 & CUMPLE \\
\hline & & HA-17,5 - B-40O-S - HP-30 & 1 & B.2.3. & 10.151 & 1,50 & CUMPLE \\
\hline & & & 2 & B.2.4. & 11.681 & 1,73 & CUMPLE \\
\hline & & HA-17.5 - B-40O-S - HP-4O & 1 & B.2.5. & 10.344 & 1,53 & CUMPLE \\
\hline & & & 2 & B.2.6. & 11.904 & 1,76 & CUMPLE \\
\hline & & HA-30 - B-50O-SD - HP-4O & 1 & B.2.7. & 13.071 & 1,94 & CUMPLE \\
\hline & & & 2 & B.2.8. & 14.715 & 2,18 & CUMPLE \\
\hline & & HA-17,5 - B-40O-S - HP-40 & 1 & B.3.1. & 8.367 & 1,24 & - NO - \\
\hline & & & 2 & B.3.2. & 9.791 & 1,45 & CUMPLE \\
\hline & & HA-30 - B-50O-SD - HP-4O & 1 & B.3.3. & 10.660 & 1,58 & CUMPLE \\
\hline & & & 2 & B.3.4. & 12.465 & 1,85 & CUMPLE \\
\hline \multicolumn{5}{|c|}{$\begin{array}{l}\text { Forjados de } 30 \mathrm{~cm} \text { de canto } \\
\end{array}$} & \multirow{2}{*}{$\begin{array}{c}\text { Carga a la } \\
\text { flecha activa } \\
\text { admisible } \\
\left(\mathbf{N} / \mathbf{m}^{2}\right)\end{array}$} & \multirow[b]{2}{*}{$\begin{array}{l}\text { Factor de Carga } \\
\mathbf{Q}_{\text {fl.act.adm. }} / \mathbf{Q}_{\text {norma }}\end{array}$} & \multirow[b]{2}{*}{$\begin{array}{l}\text { Prueba de } \\
\text { Carga EHE }\end{array}$} \\
\hline Cuadrante & $\begin{array}{c}\mathrm{N}^{\circ} \\
\text { viguetas } \\
\text { afectadas }\end{array}$ & $\begin{array}{c}\text { Materiales } \\
\begin{array}{c}\text { (h.in situ - acero - } \\
\text { h.pretensado) }\end{array} \\
\end{array}$ & $\begin{array}{l}\text { Niveles } \\
\text { tendones } \\
\text { sanos }\end{array}$ & Caso & & & \\
\hline \multirow{10}{*}{2} & \multirow{6}{*}{3} & HA-10 - B-40O-S - HP-4O & 1 & C.1.1. & 9.294 & 1,24 & - NO - \\
\hline & & & 2 & C.1.2. & 10.716 & 1,43 & CUMPLE \\
\hline & & HA-17,5 - B-40O-S - HP-40 & 1 & C.1.3. & 10.710 & 1,43 & CUMPLE \\
\hline & & & 2 & C.1.4. & 12.661 & 1,69 & CUMPLE \\
\hline & & HA-30 - B-50O-SD - HP-4O & 1 & C.1.5. & 13.283 & 1,77 & CUMPLE \\
\hline & & & 2 & C.1.6. & 15.218 & 2,03 & CUMPLE \\
\hline & & HA-17,5 - B-40O-S - HP-40 & 1 & C.2.1. & 8.775 & 1,17 & - NO - \\
\hline & Todac & & 2 & C.2.2. & 9.835 & 1,31 & CUMPLE \\
\hline & Todas & HA-30 - B-50O-SD - HP-40 & 1 & C.2.3. & 10.857 & 1,45 & CUMPLE \\
\hline & & & 2 & C.2.4. & 12.466 & 1,66 & CUMPLE \\
\hline & & HA-1O - B-40O-S - HP-4O & 1 & D.1.1. & 10.459 & 1,39 & - NO - \\
\hline & & & 2 & D.1.2. & 11.852 & 1,58 & CUMPLE \\
\hline & & HA-17,5 - B-40O-S - HP-40 & 1 & D.1.3. & 12.779 & 1,70 & CUMPLE \\
\hline & 3 & & 2 & D.1.4. & 14.183 & 1,89 & CUMPLE \\
\hline & & HA-30 - B-50O-SD - HP-40 & 1 & D.1.5. & 15.814 & 2,11 & CUMPLE \\
\hline 4 & & & 2 & D.1.6. & 17.675 & 2,36 & CUMPLE \\
\hline & & HA-17,5 - B-40O-S - HP-4O & 1 & D.2.1. & 9.917 & 1,32 & - NO - \\
\hline & Todas & & 2 & D.2.2. & 11.700 & 1,56 & CUMPLE \\
\hline & Todas & HA-30 - B-50O-SD - HP-40 & 1 & D.2.3. & 12.960 & 1,73 & CUMPLE \\
\hline & & & 2 & D.2.4. & 14.862 & 1,98 & CUMPLE \\
\hline
\end{tabular}




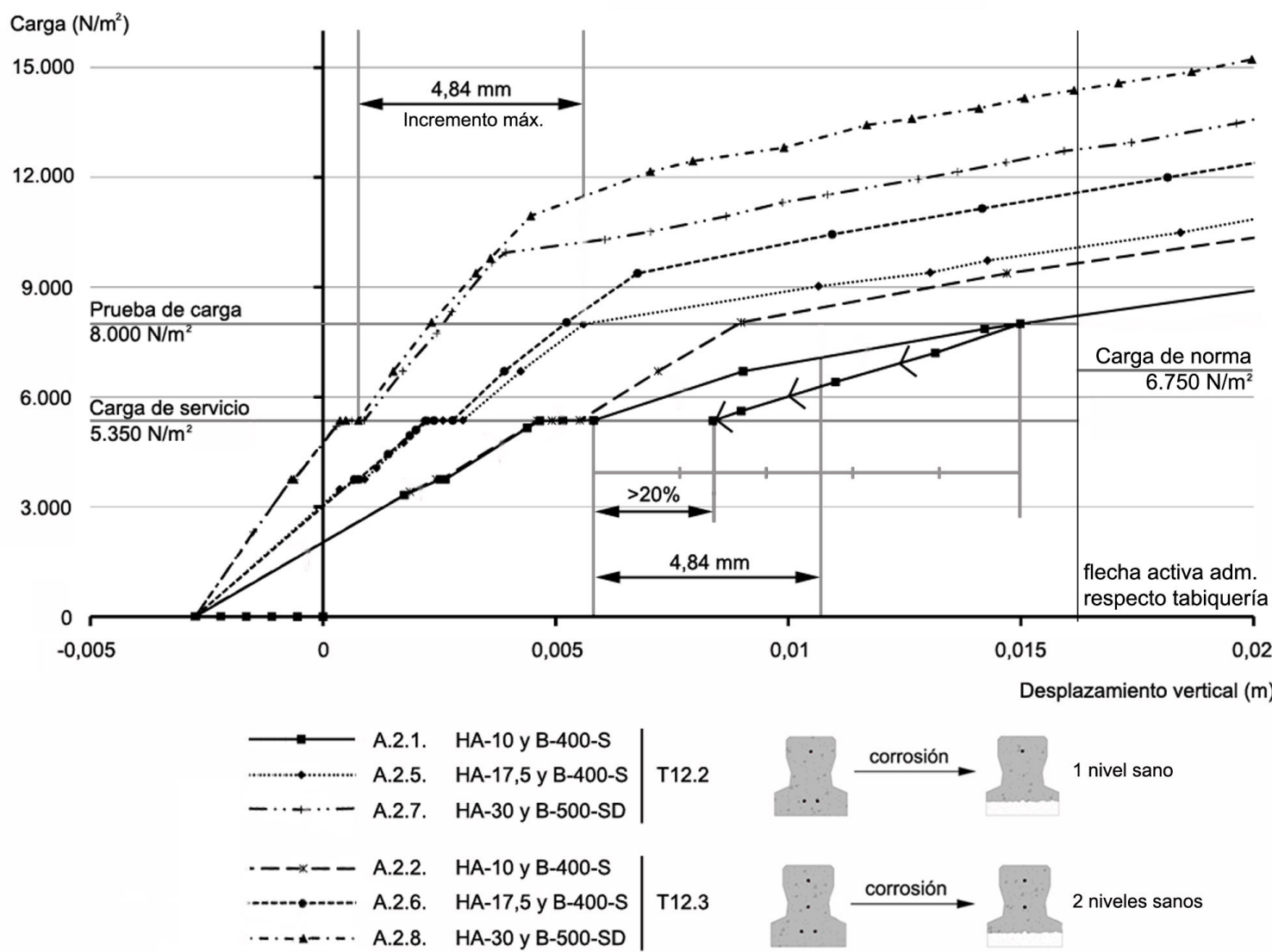

Figura 6. Curvas carga-desplazamiento vertical de los casos de 3 viguetas afectadas en el cuadrante 2 para los forjados de $25 \mathrm{~cm}$ de canto.

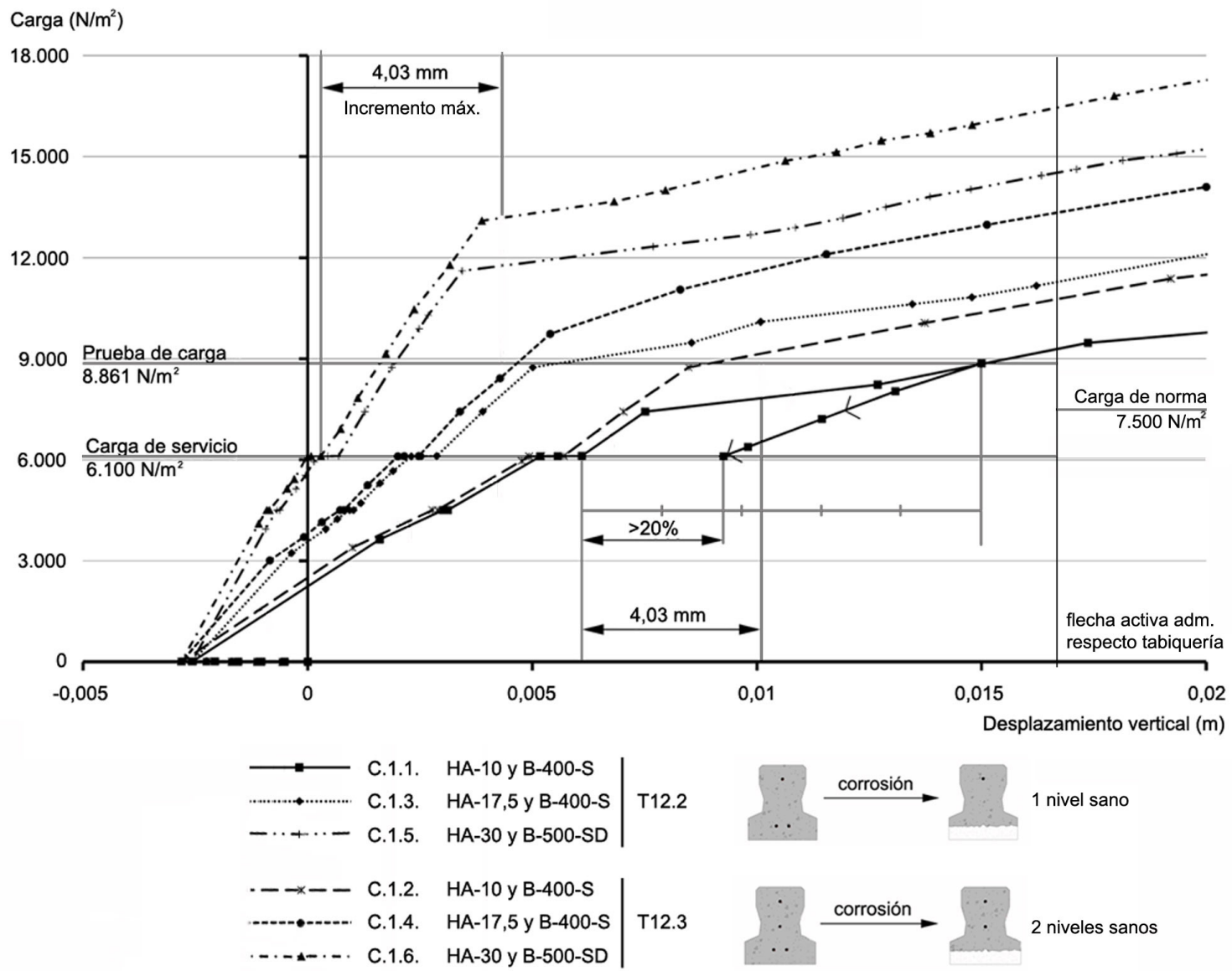

Figura 7. Curvas carga-desplazamiento vertical de los casos de 3 viguetas afectadas en el cuadrante 2 para los forjados de $30 \mathrm{~cm}$ de canto. 
recuperación de la deformada ha sido inferior a la necesaria de 4/5 partes, no cumpliendo por tanto este criterio de aceptación.

- A nivel de ejemplo, se acota también la carga a la flecha activa admisible respecto de la tabiquería para el caso más deteriorado, con lo que se obtiene el Factor de Carga.

Tal como hemos observado en los casos reales, cuando existe una sola vigueta aislada con daños, en todos los casos cumple el criterio de aceptación de estructuras existentes, y presenta valores del Factor de Carga elevados, que con materiales sanos son cercanos a 2 .

Los casos en los que la corrosión ha afectado hasta tres viguetas contiguas cumplen la prueba de carga de la EHE, excepto aquéllas en las que solo queda un nivel de tendones sanos y además los materiales posean muy bajas resistencias (casos A.2.1., B.2.1., C.1.1. y D.1.1.).

En estos casos en que no cumple se ha superado el incremento de deformada permitido, y por lo tanto se ha simulado la descarga (tramo con flechas en las Figuras 6 y 7). Al no recuperarse el $80 \%$ de la deformación, tampoco cumplen el segundo criterio de aceptación.

Llama la atención que incluso con todas las viguetas de un paño corroídas, la simulación de la prueba de carga cumple los criterios de aceptación cuando quedan dos niveles de tendones sanos, incluso con bajas resistencias de materiales. Con un solo nivel de tendones sano solo algún caso muy singular todavía cumple.

El Factor de Carga aceptable en los casos de rehabilitación es responsabilidad del experto que realiza el proyecto, y depende de las normativas locales. De todas formas, parece sensato asumir que en los casos en los que el Factor de Carga posee un valor inferior a 1,50, aunque cumpla la prueba de carga, es necesario realizar una reparación intensa. Y si el valor del Factor de Carga está próximo a 2,00, o incluso lo supera, es suficiente con una intervención ligera de reparación y protección para que el daño no continúe avanzando.

\section{CONCLUSIONES}

Se ha comprobado que el método propuesto obtiene resultados acordes con los estudios de otros autores y con la gran variedad de casos patológicos reales observados, en los que aparece corrosión en el nivel inferior de tendones de las viguetas de un forjado. Este método es extrapolable a cualquier caso distinto a los analizados en este trabajo, como pueden ser los casos en que aparezca corrosión en el refuerzo a negativos de las viguetas o que la geometría sea singular.

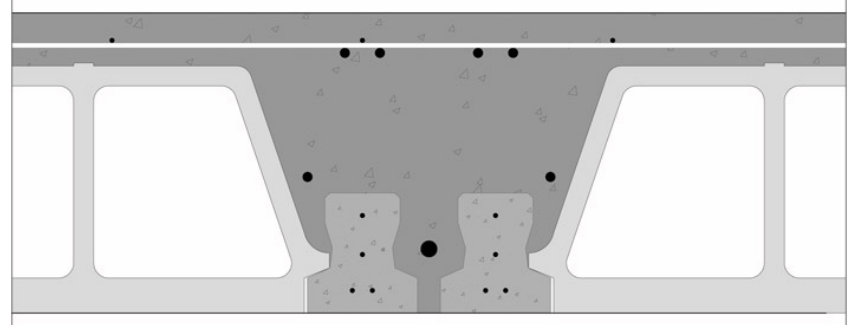

Figura 8. Detalle constructivo propuesto en las viguetas que enfrentan pilares.
En los casos en que el cortante no sea determinante, por haber realizado un estudio previo para descartar este tipo de rotura frágil, y tengamos garantizado que la rotura la determina la flexión, se puede concluir que:

- Cuando la corrosión aparece en una única vigueta, en cualquier posición del forjado, no existe un peligro inminente. Es el caso más frecuente debido a daños físicos o a humedades muy puntuales. En estos casos se cumple la prueba de carga EHE y se poseen valores de Factor de Carga superiores a 1,50, incluso con unos materiales de baja calidad y viguetas en las que únicamente queda un nivel de tendones sano al desaparecer el nivel inferior. Aquí es recomendable consolidar la vigueta y aplicarle un tratamiento de protección.

- Los casos simulados con corrosión severa de hasta 3 viguetas contiguas, también muy habituales debido a fugas continuadas en bajantes, normalmente cumplen los criterios que dicta la EHE, a excepción de los casos en que solo quede un solo nivel de tendones sanos y en que los materiales están muy degradados (casos A.2.1., B.2.1., C.1.1. y D.1.1.), con resistencias residuales del entorno de $10 \mathrm{MPa}$.

En todos los casos el Factor de Carga indica que se puede acometer la rehabilitación sin necesidad de medidas excepcionales de apuntalamiento o desalojo. La presencia de 2 niveles de tendones sanos después de la corrosión garantiza unos valores de FC superiores a 1,50, y que rara vez alcanza 2, que es considerado un valor mínimo para la aceptación por muchos autores.

La intervención necesaria puede consistir en unas medidas livianas de restauración estructural consistentes en adherir por la cara inferior unas bandas de fibra protegidas.

- Y si la corrosión severa afecta a todos los nervios de un paño es aconsejable realizar un apeo inmediato y una reparación generalizada. Esta reparación puede realizarse mediante un doblado de viguetas metálicas por la cara inferior del forjado, o también mediante bandas de fibra con un estudio específico para que quede perfectamente garantizada su adherencia.

Una conclusión muy importante obtenida en este estudio es que el fallo de las viguetas que enfrentan pilares son determinantes para el colapso total de los forjados. Mientras estas viguetas son capaces de resistir, el forjado va deformando en forma de recuadros independientes. En este tramo de las curvas carga-desplazamiento se posee una pendiente con relativa seguridad.

Cuando fallan los nervios que enfrentan pilares los paños inician un mecanismo de rotura en artesa global y la curva carga-desplazamiento cambia de pendiente bruscamente. De aquí surge una recomendación constructiva, que es la de reforzar la armadura inferior de las viguetas aproximadamente enfrentadas a pilares con redondos gruesos y bien anclados que sobrepasen los ejes de los pilares.

Esta recomendación es favorable para la resistencia en los casos de corrosión, pero también frente al fuego o al sismo, por lo que se plantea incluso para que se adopte como criterio de diseño en la edificación de nueva planta (Figura 8).

En los casos de forjados de viguetas prefabricadas este refuerzo se puede conseguir doblando la vigueta y colocando un redondo de mayor diámetro entre las viguetas. 
Siempre que aparece un daño en una estructura es necesario intervenir, aunque únicamente sea por condicionantes sociales. Este estudio permite evaluar la seguridad residual.
Según la magnitud del daño, la intervención puede ir desde unas medidas de protección para evitar que el daño continúe, hasta un refuerzo integral de toda la estructura.

\section{REFERENCIAS}

(1) Morán-Cabré, F. (1994). Estimación de la seguridad residual en estructuras de hormigón con problemas patológicos. Informes de la Construcción, 46(434): 39-51, doi: http://dx.doi.org/10.3989/ic.1994.v46.i434.1107.

(2) Giménez, E. (2007). Estudio experimental y numérico de soportes de hormigón armado reforzados con perfiles metálicos sometidos a esfuerzos de compresión simple (Tesis Doctoral). Valencia: Universidad Politécnica de Valencia.

(3) Vieitez, J.A., Ramírez, J.L. (1984). Patología de la Construcción en España: Aproximación Estadística. Resumen de Tesis Doctoral. Informes de la Construcción, 36(364): 5-15, doi: http://dx.doi.org/10.3989/ic.1984.v36.i364.1901.

(4) Instituto Valenciano de la Edificación (IVE). (2008). Guía para la Inspección y Evaluación Preliminar de estructuras de hormigón en edificios existentes. Serie guías de la calidad. Valencia: Generalitat Valenciana - Conselleria de Medi Ambient, Aigua, Urbanisme i Habitatge.

(5) Instituto Valenciano de la Edificación (IVE). (2008). Experiencia en Inspección de estructuras en edificios. Comunidad Valenciana 1991-2008. Serie guías de la calidad. Valencia: Generalitat Valenciana - Conselleria de Medi Ambient, Aigua, Urbanisme i Habitatge.

(6) Grupo Español del Hormigón (GEHO). (1994). Reparación y refuerzo de estructuras hormigón. Guía FIP de buena práctica. Boletín GEHO, 14. Madrid.

(7) Di Evangelista, A., De Leonardis, A., Valente, C., Zuccarino, L. (2011). Design and testing of corrosion damaged prestressed concrete joists: the Pescara Benchmark. En 9th International Conference on Damage Assessment of Structures (DAMAS 2011), Journal of Physics: Conference Series 305.

(8) Coronelli, D., Gambarova, P. (2004). Structural assessment of corroded reinforced concrete beams: modeling guidelines. Journal of Structural Engineering, 130(8): 1214-1224, doi: http://dx.doi.org/10.1061/(ASCE)07339445(2004)130:8(1214).

(9) Foster, S.J., Bailey, C.G., Burgess, I.W., Plank, R.J. (2004). Experimental behavior of concrete floor slabs at large displacements. Engineering Structures, 26(9): 1231-1247, doi: http://dx.doi.org/10.1016/j.engstruct.2004.04.002.

(10) Prevalesa, S.L. (2008). Ficha de características técnicas - según EHE-o8 - del forjado de viguetas pretensado modelo DITECO T12. Valencia.

(11) Ministerio de Vivienda. (2006, 28 de marzo). Código Técnico de la Edificación (CTE). Boletín Oficial del Estado, nº 74: 11816-11831. Madrid, España.

(12) Bangash, M.Y.H. (1989). Concrete and concrete structures: Numerical modeling and applications. London - New York: Elsevier Applied Science.

(13) Willam, K.J., Warnke, E.D. (1975). Constitutive model for the triaxial behavior of concrete. En Proceedings of the International Association for Bridge and Structural Engineering, ISMES, 19: 1-30. Bergamo, Italy.

(14) Vercher, J. (2013). Seguridad residual en los forjados con corrosión severa (Tesis Doctoral). Valencia: Universidat Politècnica de València.

(15) Cubel, F., Más, A., Vercher, J., Gil, E. (2012). Design and construction recommendations for brick enclosures with continuous air chamber. Construction and Building Materials, 36: 151-164, doi: http://dx.doi.org/10.1016/j.conbuildmat.2012.04.128.

(16) Brencich, A., de Felice, G. (2009). Brickwork under eccentric compression: Experimental results and macroscopic models. Construction and Building Materials, 23(5): 1935-1946, doi: http://dx.doi.org/10.1016/j.conbuildmat.2008.09.004.

(17) Dilrukshi, K.G.S., Dias, W.P.S., Rajapakse, R.K.N.D. (2010). Numerical modelling of cracks in masonry walls due to thermal movements in an overlying slab. Engineering Structures, 32(5): 1411-1422, doi: http://dx.doi.org/10.1016/j. engstruct.2010.01.019.

(18) Fanning, P. (2001). Nonlinear models of reinforced and post-tensioned concrete beams. Electronic Journal of Structural Engineering, 2: 111-119.

(19) Tavio, T., Tata, A. (2009). Predicting nonlinear behavior and stress-strain relationship of rectangular confined reinforced concrete columns with Ansys. Civil Engineering Dimension, 11(1): 23-31.

(20) Rodriguez, J., Ortega, L.M., Casal, J. (1997). Load carrying capacity of concrete structures with corroded reinforcement. Construction and Building Materials, 11(4): 239-248, doi: http://dx.doi.org/10.1016/So950-0618(97)ooo43-3. 